\title{
Employment Interview Screening: Time to Face the Ink
}

\author{
Paul J. Antonellis, Jr., Ed.D. \\ Faculty \& Coordinator of Human Resource Management Program, \\ Merrimack College, North Andover \\ USA \\ Rachel Silsbee, M.Ed \\ Assistant Director of Student Success: Writing Center \\ Merrimack College, North Andover \\ USA
}

\begin{abstract}
This article focuses on the impact that face and/or neck tattoos may have on the employment screening process. With some estimates placing the number of possible candidates with tattoos as high as $28 \%$, and the increase in social acceptance of tattoos, reveals that this research may play a significant role for the employer and the candidate during the employment screening process. Tattoos today can be found on men and women, with no limits on professional status and the tattoo designs can hold profound meaning to the wearer. This qualitative study analyzed data from 243 participants. The data was broken down into gender responses when it comes to assessing a candidate with a face/neck tattoo during the employment screening process. One significant theme that emerged is that $50 \%$ of the participants reported that they would hire a candidate with a visible face/neck tattoo depending on the position, revealing that employment screeners still feel that a visible tattoo may be acceptable for some positions and not acceptable for other positions.
\end{abstract}

Keywords: Interview, Tattoo, Selection, Employment Screening

\section{Introduction}

A great deal of research in the field of Human Resource Management, Industrial-Organizational Psychology and Social Psychology has focused on the impact that tattoos have in the employment arena when it comes to bias, stigma, prejudice, visual cues, social class, recruitment, taste-based discrimination, and/or attitudes (Burgess \& Clark, 2010; Larson, Patterson \& Markham, 2014; Mendez, 2016; Miller, Nicols \& Eure, 2009; Seiter \& Hatch, 2005;Swanger, 2006;Totten, Lipscomb, \& Jones 2009). One area of tattoo research with limited literature includes what extent does a neck and/or face tattoo have on the Employment Screening Process (ESP) (Timming, Nickson, Re, \& Perrett, 2017; Zestcott, Bean, \& Stone, 2017). Face and neck tattoos are challenging at best to cover up during an employment interview; this may result in the interviewer seeing the face or neck tattoo compared to a tattoo located on the upper arm that can be covered up with a dress shirt, leading to the tattoo being hidden from view during the Employment Screening Process (ESP).In their study, Dillingh, Kooreman, and Johannes (2016) reported that $7.4 \%$ of the participants reported having a tattoo on the head/face and $2.3 \%$ of participants reported that they had a tattoo on the neck area $(n=512)$. Ellis $(2015)$ and other researchers have suggested that exposed tattoos can negatively influence the ESP(Burgess \& Clark, 2010).Whorton (2015) acknowledged that exposed tattoos coupled with the employment screener's personal bias or stigma may result in a negative evaluation of the candidate.

The wide spread acceptance of tattoos from a social standpoint, in conjunction with the increasing number of people who have one or more tattoos, has been rising over the past few years (Dickson, Dukes, Smith \& Strapko, 2015; Laumann \& Derick, 2006; Walzer \& Sanjurjo, 2016).As the number of people receiving tattoos today increases, the research supports the idea that the acceptance rate of tattoos is becoming more widely accepted by people (McBride, 2017). Ruffle and Wilson (2017) reported that 40\% of Americans under the age of 40 have one or more tattoos revealing that the possible pool of candidates for employment may be tattooed $(\mathrm{n}=512)$.Swami, Tran, Kuhlmann, Stieger, Gaughan, and Voracek, (2016) claimed "tattooed and non-tattooed adults nowadays are more similar than different" (p.42). 
The evidence supports the idea that today we are more likely to interview a person with a tattoo; therefore, it is important that employers and people with tattoos recognize how tattoos can influence the ESP.

\subsection{Statement of the Problem}

Based on the increase in social acceptance of tattoos and the evidence of bias when it comes to visible tattoos during the ESP, the goal of this article is to explore to what degree, if any, does a neck and/or face tattoo have on the ESP. Current research places the estimated range of U.S. employment applicants with one or more tattoos in the range of $16 \%$ to as high as 28\% (Larson, Patterson, \& Markham, 2014; Laumann \& Derick, 2006). A 2015 Harris Poll $(\mathrm{n}=2,225)$ reported that Millennials $(47 \%)$ and Gen Xers $(36 \%)$ have one tattoo, indicating that the percentage of possible employee candidates having a tattoo may in fact be higher than originally reported in prior studies(Shannon-Missal, 2016). The estimates of people with one or more tattoos have been reported from $8 \%$ to $10 \%$ in Germany, France, Finland, and Australia (Kluger, 2015). It is projected that this range will continue to increase over time based on the widespread acceptance of tattoos today.

The goal of this article is to provide employers and employment candidates with insight as to the impact that face/neck tattoos may have during the ESP in the United States. The results of this research may provide employers and human resource management professionals with additional information on the increase in candidates with tattoos and the steps they can take to prevent possible negative ratings of candidates during the ESP. The results of this research may prove helpful to those candidates with one or more tattoos who are or may be participating in an ESP and the steps they can take to prevent possible negative evaluations during the ESP. The results of this research may provide an avenue to discuss the impact that tattoos have on the screening process of candidates and that the results may be incorporated in the employer training program used to train screeners.

\section{Theoretical Underpinning}

\subsection{Historical Factors}

The topic of tattoos is not new by any means; in fact, some have traced the history of tattoos on the body to Egyptian mummies around 400 B.C. (Ferguson-Rayport\& Straus, 1995; Laumann, \& Kluger, 2018; Levy, Sewell, \& Goldstein, 1979; Sperry, 1991). In the past, tattoos have had an association to deviant behaviors, gangs, and criminal behavior. Biernant and Dovido (2003) pointed out that the prison population is often covered in multiple tattoos, and the association between crime and tattoos is in part based on this stereotype of the prison population. Kluger (2015) asserted that the prevalence rates range for the prison population having a tattoo from $9 \%$ to a high of $70 \%$ depending on the location of the prison. The practice of tattooing while incarcerated remains illegal; however, the practice of make shift tattooing of fellow inmates remains a common practice. One researcher estimates that about $40 \%$ of all inmates have received one or more tattoos while incarcerated (Kluger, 2015). Based on the evidence presented, society's belief that people with tattoos are associated with criminal behavior is supported.

Research studies have asserted the connection between deviant behavior and people with tattoos. Deviant behavior is often classified as binge drinking, having multiple sexual partners, murders, suicide, and accidental death (Blackburn, Cleveland, Griffin, Davis Lienert, \& McGwin, 2012; Koch, Roberts, Armstrong, \& Owen, 2010). The literature supports the assumption that people generally feel that tattoos are associated with deviant behavior and as a result any person with tattoos must be engaging in this same behavior. Between the ' $50 \mathrm{~s}$-' $70 \mathrm{~s}$ society witnessed a shift in opinions about tattoos, from tattoos once accepted as a form of fraternal members (military, firefighters, law enforcement)to tattoos being associated with deviant behavior (Serup, 2015). This belief may be held by the person who is conducting the ESP resulting in a negative impact for the candidate with a tattoo.

From a historical standpoint, tattoos have been traced back to certain religious rights of passages for Hawaiians, Samoans, and Tahitians and have nothing to do with deviant behaviors, gangs, and criminal behaviors. A noteworthy history of tattoos can be found in the military, which allowed members of the special group to be easily identified by their membership in a branch of the military or a specialized unit. Some military personnel may have a tattoo that is honoring a fallen member of the unit or memorializing the time they spent in a given country during war time. Military personnel may use tattoos to memorialize the events that they have been exposed to or to make sense of the duties they performed during wartime (Dyvik \& Welland, 2018; Sanders, 1988). Religiously affiliated tattoos and military tattoos have significant meaning to the tattooed individual. 


\subsection{Tattoo Location}

The tattoo location, size, color, text, and design can all have meaning to the individual. Some believe tattoos can influence the "energy field" of the individual and have a positive impact on the individual's self-worth. The location of the tattoos can also provide insight to the individual's personality, beliefs, or orientation (Barretta, 2017; Wilson, Wilson, \& Wilson, 2017). The reasons a person may get a tattoo and the design is beyond the scope of this research, but the reasons are countless.

Facial tattoos have been shown to be a significant factor in making judgements about a person with a tattoo (Ball \& Elsner, 2017; Funk \& Todorov, 2013; Swami, Stieger, Pietschnig, Voracek, Furnham, \&Tovee, 2012). The focus of this research is to build upon this prior knowledge as to the impact that facial tattoos may have on the employment screening process.

Dillingh, Kooreman, and Potters (2016) research observed that $10 \%$ of the participants had tattoos on the face and neck area of the body.The decision to have a tattoo placed on the face or neck area has been shown to have a negative economic and social consequence for the individual. Brallier, Maguire, Smith,\& Palm (2011) suggested that people in a managerial capacity and the greater parts of society feel that a visible tattoo (Arndt, McCombs, Tolle, \& Cox, 2017) remains a factor in determining suitability for employment.

Visible tattoos can have a negative influence in the ESP (Foltz, 2014). Dickson, Duke, Smith, and Strapko (2015) claimed that face, neck, and hands tattoos are considered to be improper in a majority of employment positions. This would tend to support the ideas of Ball and Elsner (2017) and Funk and Todorov (2013) that face tattoos can influence a person's judgement of the tattooed person. In many cases, it is difficult, if not impossible, to cover up a face, neck, or hand tattoo which would leave the tattoo visible during the ESP. The tattoo location may allow for easy concealment in some cases during the ESP,thus preventing the employment screener from seeing the tattoo and not negatively impacting the screening. Foltz (2014) and Timming, Nickson, Re, andPerrett(2017) observed that people with visible tattoos were hired less often than employee candidates with no visible tattoos.

\subsection{Stereotypes}

Steele (1997) suggested that a stereotype could be defined as a sub-group of people who all have the same negative image or idea on a given person or topic. As witnessed by prior stereotypes held by society, people with tattoos are viewed as participating in deviant behaviors, being part of a gang, or former prisoner (Blackburn, Cleveland, Griffin, Davis Lienert, \& McGwin, 2012; Koch, Roberts, Armstrong,\& Owen, 2010). Funk and Todorov (2013) argued that a face tattoo that is visible during a courtroom setting affected judgment of guilt. One research study suggests today people with tattoos and people without tattoos are considered equally trusted (Ruffle \& Wilson, 2018) suggesting that the stereotype of people with tattoos may be shifting to more acceptance. It is further suggested that as additional people receive tattoos, the old stereotypes will begin to fade.

\subsection{First Impression}

The topic of first impression is not a new area of interest in the ESP and will remain a significant area of interest going forward. To better understand the significance of first impressions, Fiske (1984) observed that negative traits provided more weight with an individual than a positive trait. Since this earlier study, additional research has been conducted that further supports this conclusion (Dougherty, Turban, \& Callender, 1994; Ito, Larsen, Smith, \& Cacioppo, 1998; Wood, 2014). Therefore, those who reveal a trait that the employment evaluator deems negative will result in an adverse rating during the employment screening process due in part to the first impression. The ESP may involve a review of a resume, a video conference call, or even a face to face interview. Macan (2009) disclosed that the employment interview is the most widely used employment screen tool next to the resume review. Given the significance of the interview process, one would argue that the factors that may influence this process should be considered. During the screening process, first impressions may influence the screening process, especially if the trait was considered as negative by the evaluator.

Macan and Dipboye (1988) believed that employment screeners who felt that the candidates' qualifications were substandard would tend to ask more difficult questions of the candidate than those they considered qualified candidates during the screening process. They further acknowledged that little evidence revealed that "preinterview" had any significance in the screening process, leading to the conclusion that first impressions can have an influence in the ESP. 
In our prior research study, Antonellis, Berry, and Silsbee (2017)found that first impressions with people during the formal interview process created a significant impression on the male rater $(77 \%)$ while female raters were pointedly less $(23 \%)$.

First impressions during the ESC may be the first time the employer screener makes face to face contact with the candidate via a video call or in-person interview. Todorov, Pakrashi, and Oosterhof (2009) insisted that perceptions of a face to face meeting are formed routinely and immediately by people. Sofer, Dotsch, Wigboldus, and Todorov (2015) reported that face recognition plays a role when a person is determining trustworthiness in another person. It should be pointed out that face-based first impression holds low validity rates (Olivola\& Todorov, 2010); however, people often go with the first impression. Additional research suggests that a person with a tattoo may be considered more creative and willing to express themselves, while a majority of people consider people with a tattoo to be unattractive, uncaring, and less intelligent (Mendez, 2016; Seiter \& Hatch, 2005). Considering the research that supports the idea that first impressions can play a significant role in the employment screening process, it would suggest that first impressions should be discussed openly with the employment screeners to minimize the risk of allowing first impressions to negatively impact the ESP.

\subsection{Professional/Personal Image}

The image of people with tattoos has shifted over the past 10-20 years. In today's mainstream culture, people are viewing tattoos as a form of art or expression with less people seeking the connection between tattoos and deviant behavior. The meaning and image behind today's tattooed person is far different in meaning than 30 years ago (Roberts, 2012; Sanders, 1988). Asking a millennial generation candidate to cover up his/her tattoos is like asking that person not to be themselves. Some organizations may have a company policy that require exposed tattoos to be covered up, which is most often seen with positions that deal directly with customers. The employer in the United States has a right to establish reasonable standards for tattoos, facial piercings and grooming. It is best practice to ensure that the policy does not violate Title VII of the 1964 Civil Rights Act of the United States. In this case, Federal law protects those in a protected class status from discrimination (Ellis, 2015; Mendez, 2016). Today, more and more people are receiving tattoos that they consider to identify them by gender or physical appearance, opening the door to a protected class classification (Calvasina \& Calvasina, 2017). It should be noted that simply having a tattoo and not being hired because you have a tattoo is not considered a violation of the law in the United States. Having such a policy may seem simple, but to Millennials (Gen Y) and Centennials (Gen Z) employees with tattoos may find it offensive that the company is asking them to cover up who they are and pretend to be someone different while at work.

Dickson, Dukes, Smith, and Strapko (2015) identified several reasons that people tend to get a tattoo ranging from self-expression to memorializing the significance of an event (e.g., Death, beating Cancer). The findings further support the idea that those with tattoos have significant meaning to the individual. Roggenkamp, Nicholls, and Pierre (2017) claimed,"Motivations for tattooing vary between genders, with women more likely to seek tattoos for personal decoration and to feel more independent, and men more likely to use them as symbols of group identity" (p. 151).Laumann and Derick, (2006) found that those people with one tattoo are often subtle tattoos and easily hidden, while tattooed people (with multiple tattoos) are often the ones who will have visible tattoos.

A tattoo might appear to be art to one person and may be offensive or unprofessional to the next person. A visible tattoo can be as simple as a small flower or as detailed as a large creative scene displayed on the entire back of an individual. The larger question is what is considered an offensive and/or unprofessional visible tattoo and what can be considered art on the body is beyond the scope of this research. Zestcott, Bean, and Stone, (2017) suggested that having a tattoo near the face would be considered unprofessional based on the customer opinion. Some organizations may use the verbiage that the tattoos cannot have an offensive phrase or image and the tattoos may not depict profanity or nudity. This verbiage can be considered subjective and left up to the observer to decide what is offensive and/or unprofessional. Having a policy that completely bans tattoos may open up the employer to a legal challenge if the policy does not make exceptions for tattoos that represent religious beliefs or cultural practices. The company policy should provide a detailed list of what the company considers "offensive and/or unprofessional." During the policy development, answering the following question is recommended, would the tattoo phrase or image rise to the lever that it might harm the company image or would the tattoo create a hostile work environment for other employees in the organization? (Bigu \& Anastasiu, 2016; Foltz, 2014). 
As emphasized, to prevent offensive or unprofessional tattoos may be the easy part of creating a written policy; the real challenge may be enforcing the areas that are up for debate as to offensive and/or unprofessional.

\section{Methodology}

Participants $(\mathrm{N}=243)$ for this research were collected on a social media site that is designed for professional networking. We collected the textual data provided by respondents on the Human Resource Management group discussion board. Respondents in the social media site and the Human Resource Management group are open to anyone after filling out a free membership. An original post was made in the Human Resource Management group by the principal investigator with a photo of a young male with a visible tattoo on his "face" with the following caption "What're your thoughts as an employer? Do you think tattoos are now accepted more in society or would an individual having tattoos affect your decision to hire a candidate?'Respondents from June 19 to 21 , 2017 posted textual comments on the discussion board and the principal investigator copied the textual posting into an Excel sheet with the participants posted name and gender. The raw data was only accessible to the principal investigator (Salmons, 2016, 2017).Steps were taken to ensure no duplicate postings were included in this survey or postings with the same name. In order to utilize only original respondent postings, no duplicate postings were indicated. Once the data was collected, names were removed and a numeric number was assigned to each respondent.

The research methodology selected for this research was thematic analysis of the qualitative data (Boyatzis, 1998; Braun \&Clarke, 2012; Fereday \& Muir-Cochrane, 2006; Rubin \& Rubin, 1995). In total, 243 textual postings were recorded while applying the verbatim principle, during the 3-day period. Beyond this timeframe, no additional data was collected. The respondents provided textual responses from a low of one sentence containing six words to a maximum response with 15 sentences and 314 words. Based on a review of the respondent textual postings, a majority of the respondents are predominately from the United States with a few postings making reference to other countries. We believe that the 243 respondent's textual statements provide firsthand experience and insight as it pertains to the ESP when confronted with a candidate with a face/neck tattoo. A majority of the respondents indicated that they have hired or would hire a person with a face/neck tattoo, indicating that the respondents are the people who are conducting the ESP. The principal investigator did not have any influence once the original post was made to social media, and no further comments or edits were performed. The goal was to collect first-hand statements made by people who have hiring responsibilities to allow qualitative data analysis for emerging themes to uncover possible underpinnings of the beliefs held of tattoos during the ESP currently in society. In order to maintain the respondent's voice and tone, we did not edit any of the textual comments. The ten emerging themes were based on the respondent's textual responses and are reflective of respondent's views.

\subsection{Research Question}

The purpose of the research study was to identify common themes from statements provided by people who have the responsibility of conducting the ESP and determine to what extent a face tattoo might influence the ESP. The following questions guided the study:

RQ 1: How do people who are responsible for employment interviewing feel about facial tattoos?

RQ 2: To what extent, if any, do candidates' neck/face tattoos have on the employment screening process?

The purpose for the two research questions was to guide the study and provide insight as to how employment screeners feel about visible facial tattoos during the ESP and to what extent, if any, does the tattoos have on the screener. The new knowledge supports current literature on tattoos and employment, provide a point of discussion for training of individuals who conduct the ESP, and provide knowledge to those who have a visible tattoo or those who might be considering obtaining a tattoo.

\section{Data Analysis and Findings}

\subsection{Data Analysis}

The first step included collecting all the raw data in an Excel sheet and checking for duplicate entries from respondents. The second step was to remove the names of each respondent and replace the names with a numeric value in order to protect the identity of the respondents. This process was conducted by the principal investigator. The third step involved reading each of the respondents' textual responses and reflects on the themes that seemed to be emerging. The fourth step was to review the current literature on the issue of employment screening and tattoos. 
The fifth step, which occurred a week later, involved re-reading the textual responses and beginning to write down emerging themes while attempting to use the respondents' textual words. After compiling a list of multiple themes, the goal was to narrow down the initial list to ten themes. The next step was to conduct a detailed review of the textual responses and begin to see how each of the ten themes connected to the responses provided.

In some cases, multiple themes could be identified by one response. The final step of the process included determining how the research findings supported or disproved the current literature.

\subsection{Findings}

In our research, we observed that $49(20 \%)$ of the respondents said they would not hire a candidate with a face/neck tattoo, with $24 \%$ female and $76 \%$ male respondents; of those responding, $14 \%$ did not have any tattoos themselves, $63 \%$ did not disclose if they had any tattoos, and 23\% said they had a tattoo (See Table 1). A majority of the respondents $121(50 \%)$ said that they would hire a person with a face/neck tattoo depending on the position, leading us to believe that some respondents still hold a level of bias towards individuals with tattoos on the face.

As reported, $30 \%$ of the respondents indicated that they would hire the person with a face/neck tattoo suggesting that tattoos are becoming mainstream and the acceptance rate is increasing. However, $50 \%$ of the respondents indicated that it would depend on the position, resulting in the fact that some people may be accepting, but at the same time, feelings of bias and/or stereotypes may have an influence in the decision. The decision to hire someone with a visible neck/face tattoo is aligned with the findings of Dickson, Duke, Smith, and Strapko (2015). The following reveal some of the textual data provided by participants:

"The person has made a choice to have ink on his/her face and I am also making a choice not to hire you. Bottom line, we are all allowed to make a choice, my choice is not to hire." Male. (6)

"Tattoos are a personal choice and a personal expression. Those two things don't necessarily align in/with business and professional worlds." Female. (101)

"Location, location and location matters for tattoos on your body. The tattoo on the neck or face is a big NO for me." Female. (156)

"As the employer, I can hire who I want, I will not hire a person with a face tattoo but would be willing to hire a person with a tattoo on the arm or leg." Male. (242)

Table1. Participant's willingness to hire the individual with exposed neck/face tattoo.

\begin{tabular}{lllllll}
\hline Participants Responses & $\mathbf{n}$ & $\mathbf{\%}$ & $\begin{array}{l}\text { Female } \\
\mathbf{n}\end{array}$ & $\begin{array}{l}\text { Female } \\
\mathbf{\%}\end{array}$ & $\begin{array}{l}\text { Male } \\
\mathbf{n}\end{array}$ & $\begin{array}{l}\text { Male } \\
\mathbf{\%}\end{array}$ \\
\hline Yes to hire & 73 & 30 & 21 & 29 & 52 & 71 \\
Depends on position & 121 & 50 & 36 & 30 & 85 & 70 \\
No to hire & 49 & 20 & 12 & 24 & 37 & 76 \\
\hline Total & $\mathbf{2 4 3}$ & $\mathbf{1 0 0}$ & $\mathbf{6 9}$ & $\mathbf{1 0 0}$ & $\mathbf{1 7 4}$ & $\mathbf{1 0 0}$ \\
\hline
\end{tabular}

The emerging themes from the data are listed in Table 2 with the breakdown of gender codes for each theme. The main theme emerging from this study was "First Impressions/Stigma" with the code appearing 298 times. If we break this down by gender, the code appears in $77 \%$ of male respondent textual statements and $23 \%$ of female textual statements. Having a visible face/neck tattoo provides that first impression to the screener. The following are some of the textual data provided by participants:

"It is human nature to immediately judge a person on how they look be it hair color, piercings, fashion sense etc. our unconscious bias and in many cases, this can start things on the wrong footing." Female. (149)

"We are all subjected to the "first impression" scenario. This is why I do not have any tattoos. It is a fact of life." Male. (190)

"Decisions you make about your body in what you put in it what you cover it in and how you present it will always have an influence on how people see you." Male. (234)

"Let's be honest, we live in a culture which has a split opinion over body art. Communication and first impressions is partly based on appearance and visual cues. First impressions are lasting as the basis for business relationships." Female. (236) 
However, when the ten themes are broken down by gender, male codes for "First Impression/Stigma" is the largest and female codes for "Visible Ink" is the largest, supporting the idea that a visible tattoo plays a role in the first impression during the ESP as supported by the works of Timming, Nickson, Re, and Perrett, (2017).

Table 2. Themes for social media responses to tattoos and employment interview screening.

\begin{tabular}{llllll}
\hline Themes & $\mathbf{n}$ & $\begin{array}{l}\text { Male } \\
\text { n }\end{array}$ & Percent & $\begin{array}{l}\text { Female } \\
\text { n }\end{array}$ & Percent \\
\hline First Impression/Stigma & 298 & 229 & 77 & 69 & 23 \\
Visible Ink & 270 & 192 & 71 & 78 & 29 \\
Appropriate/Acceptance & 267 & 196 & 73 & 71 & 27 \\
Image/Corporate Culture/Policy & 213 & 151 & 71 & 62 & 29 \\
Tattoo Location & 180 & 133 & 74 & 47 & 26 \\
Neck/Face/Hands & 170 & 125 & 74 & 45 & 26 \\
Talent/Qualified/Skills/Ability & 152 & 114 & 75 & 38 & 25 \\
Depends on Job/Position & 148 & 113 & 76 & 35 & 24 \\
Concealed/Covered Up & 82 & 49 & 60 & 33 & 40 \\
Offensive/Polarizing/Hate & 68 & 51 & 75 & 17 & 25 \\
\hline TOTAL & $\mathbf{1 8 4 8}$ & $\mathbf{1 3 5 3}$ & $\mathbf{7 3}$ & $\mathbf{4 9 5}$ & $\mathbf{2 7}$ \\
\hline
\end{tabular}

Table 2 shows the rank order of the ten emerging themes by codes. The first three themes are: Impression/Stigma, Visible Ink, Appropriate/Acceptance, and Image/Corporate Culture/Policy representing 56\% of the coding. Regardless of the shift in academic writing and social shift of how people today view tattooed people, we still have underpinnings of older views reported by Sander (1988) that can have a negative impact on the ESP today (Foltz, 2014; Mendez, 2016; Timming, Nickson, Re, \& Perrett, 2017).As indicated, 8\% of the coding reveals that the respondent's textual responses are in relation to a candidate's "Talent/Qualification/Skills/Ability."When responding to the posted photo of the person with a neck/face tattoo, a low number of respondents feel that talent, qualifications, skills, or ability is considered during the ESP.

\section{Discussion}

Research findings indicate that $20 \%$ of the participants would not hire a person with a neck/face tattoo, while $50 \%$ of the participants revealed that it would depend on the position if they would hire a person with a neck/face tattoo. The findings are consistent with Antonellis, Berry, and Silsbee (2017), Ellis (2015), Foltz (2014), Dickson, Duke, Smith, and Strapko (2015), and Arndt, McCombs, Tolle, andCox (2017) whose findings exposed that tattoos can have a negative impact on the ESP.

The first emerging theme revealed that the participant's words or phrases indicate "First Impression/Stigma" relates to the neck/face tattoos during the ESP. This theme supports the work of Todorov, Pakrashi, and Oosterhof (2009) in that people use perceptions of a person when they first meet the person. This finding would suggest that the first time that the employment screener meets a candidate for an interview (in person or video) that first impressions and/or the stigma of having a tattoo are critical elements in the ESP. The second emerging theme was "Visible Ink" revealing that participant's words or phrases indicate that a candidate who has a visible tattoo may have a negative impact on the ESP. The top gender difference, when it comes to "First Impression/Stigma," reveals $77 \%$ of male words or phrases when considering neck/face tattoos, where as "Concealed/Cover Up" reveals $40 \%$ of female words or phrases when considering neck/face tattoos during the ESP. Our findings support the literature that suggests that during the ESP, visible tattoos can have a negative impact on the screening process as it pertains to the area of bias (Arndt, McCombs, Tolle, \& Cox, 2017; Larson, Patterson, \& Markham, 2014; Whorton, 2015).

It is our recommendation that employers consider the findings in this research when developing ESP training programs. The issue of first impressions and tattoo stigma should be reviewed, along with the steps to minimize negative first impressions and visible tattoo stigma or bias. In addition, the findings support the idea that the organization should review the company policy to ensure that it reflects current trends when it comes to corporate image and culture. 


\section{Conclusion}

The current study has attempted to provide insight into the ESP as it pertains to what impact does a face/neck tattoo have during the process. Given the fact that some estimates suggest that more than $28 \%$ of candidates for current jobs will have a minimum of one or more tattoos, attention should be given to this topic. The research will provide new information to the field of human resource management, employment screeners, and people who may have or may be considering a tattoo and the possible negative impact that a face/neck tattoo has on the ESP and long-term economic losses. The information provided in this study may also provide the employer with an opportunity to review current ESP and current policies that may place the organization in legal jeopardy.

While this research has some limitations, we can only assume that the respondents have provided truthful textual data as it pertains to the ESP and neck/face tattoos. We also acknowledge that the respondents written statements could be influenced by the fact that written statements was posted with the respondents name on the social media site. Our recommendation would be to expand the number of participants and to conduct detailed qualitative samplings to gain a deeper understanding of the responses of participants who have direct responsibility for the ESP.

\section{References}

Antonellis, P., Berry, G., \& Silsbee, R. (2017). Employment Interview Screening: Is The Ink Worth It?Global Journal of Human Resource Management, 5(2), 38-53.

Arndt, A. D., McCombs, G., Tolle, S. L., \& Cox, C. (2017). Why Are Health Care Managers Biased Against Hiring Service Providers With Tattoos?Services Marketing Quarterly, 38(2), 88-99.

Ball, J., \& Elsner, R. J. (2017). Changes in Aesthetic Appeal of Tattoos Are Influenced by the Attractiveness of Female Models. Psychology, 7(4), 247-252.

Barretta, L. (2017). Conscious Ink: The Hidden Meaning of Tattoos: Mystical, Magical, and Transformative Art You Dare to Wear. Red Wheel/Weiser.

Bigu, D., \&Anastasiu, I. (2016). Employee's Life Outside The Workplace: Ethical Challenges of Privacy. In Proceedings of the International Management Conference, 10(1), 520-525.

Blackburn, J., Cleveland, J., Griffin, R., Davis, G.G., Lienert, J., McGwin, G. (2012). Tattoo frequency and types among homicides and other deaths, 2007-2008. A matched case-control study. American Journal of Forensic Medicine and Pathology. 33(3), 202-205. doi:10.1097/PAF.0b013e318221b67d

Boyatzis, R. E. (1998). Transforming qualitative information: Thematic analysis and codedevelopment. Thousand Oaks, CA: Sage.

Brallier, S. A., Maguire, K. A., Smith, D.A., \& Palm, L. J. (2011). Visibletattoos and employment in the restaurant industry. International Journal of Business and Social Science, 2(6), 72-76.

Braun, V., \&Clarke, V.(2012). Thematic analysis. APA Handbook of Research Methods in Psychology, Vol. 2., APA. 57-71.DOI: 10.1037/13620-004

Burgess, M., \& Clark, L. (2010). Do the "savage origins" of tattoos cast a prejudicial shadow on contemporary tattooed individuals. Journal of Applied Social Psychology, 40, 746-764.

Calvasina, G. E., \& Calvasina, R. V. (2017). Employer appearance and grooming standards: Legal, policy, \& practice issues involving tattoos and body piercings. Proceedings Of The American Society Of Business \& Behavioral Sciences, 24136.

Dickson, L., Dukes, R. L., Smith, H., \&Strapko, N. (2015). To ink or not to ink: The meaning of tattoos among college students. College Student Journal, 49(1), 106-120.

Dillingh, R., Kooreman, P., \& Potters, J. J. (2016). Tattoos, life style and the labor market. IZA Discussion Paper No. 9675. Available at SSRN:https://ssrn.com/abstract=2725036

Dougherty, T. W., Turban, D. B., \&Callender, J. C. (1994). Confirming first impressions in the employment interview: A field study of interviewer behavior. Journal of Applied Psychology, 79(5), 659.

Dyvik, S. L., \&Welland, J. (2018). 'War Ink': sense-making and curating war through military tattoos. International Political Sociology.

Ellis, A. D. (2015). A picture is worth one thousand words: Body art in the workplace. Employment Responses Rights Journal, 27(2), 101-113. DOI: 10.107/s10672-014-9254-1 
Fereday, J., \& Muir-Cochrane, E. (2006). Demonstrating rigor using thematic analysis: A hybrid approach of inductive and deductive coding and theme development. International Journal of Qualitative Methods, 5(1), 80-92.

Ferguson-Rayport, S. M., Griffith, R. M., \& Straus, E. W. (1995). The psychiatric significance of tattoos. Psychiatric Quarterly, 29, 112-131. DOI: 10.1007/BF01567443

Foltz, K. A. (2014). The Millennial's perception of tattoos: self expression or business faux pas? College Student Journal, 48(4), 589-602.

Funk, F., \& Todorov, A. (2013). Criminal stereotypes in the courtroom: Facial tattoos affect guilt and punishment differently.Psychology, Public Policy, and Law, 19(4), 466-478.

Ito, T. A., Larsen, J. T., Smith, N. K., \& Cacioppo, J. T. (1998). Negative information weighs more heavily on the brain: the negativity bias in evaluative categorizations. Journal of Personality and Social Psychology, $75(4), 887$.

Kluger, N. (2015). Epidemiology of tattoos in industrialized countries. In Tattooed skin and health (Vol. 48, pp. 6-20). Karger Publishers.

Koch, J. R., Roberts, A. E., Armstrong, M. L, \& Owen, D. C. (2010). Body art, deviance, and American college students. The Social. 47(1), 151-161. doi:10.1016/j.soscij.2009.10.001

Larson, G., Patterson, M., \& Markham, L. (2014). A deviant art: Tattoo-related stigma in an era of commodification. Psychology and Marketing, 31(8), 670-681.

Laumann, A. E., \& Derick, A. J. (2006). Tattoos and body piercings in the United States: A national data set. Journal of the American Academy of Dermatology, 55(3), 413-421.

Laumann, A. E., \& Kluger, N. (2018). History and Epidemiology of Tattoos and Piercings: Legislation in the United States and in Europe. In Dermatologic Complications with Body Art (pp. 1-19). Springer, Cham.

Levy, J., Sewell, M., \& Goldstein, N. (1979). II. A short history of tattooing. The Journal of Dermatologic Surgery and Oncology, 5(11), 851-856.

Macan, T. (2009). The employment interview: A review of current studies and direction for future research. Human Resource Management Review. 19(3), 203-218.

Macan, T. M., \&Dipboye, R. L. (1988). The effects of interviews' initial impression on information-gathering. Organizational Behavior and Human Decision Processes, 42(3), 364-387.

Mendez, M. (2016). Attitudes toward Tattoos in the Work Place. Angelo State University Social Sciences Research Journal, 2(2).

McBride, D. L. (2017). Clinical Guidance to Tattooing and Piercing Among Youth. Journal of Pediatric Nursing.

Miller, B. K., Nicols, K. M., \&Eure, J. (2009). Body art in the workplace: Piercing and prejudice? Personnel Review, 38(6), 621-640.

Olivola, C. Y., \& Todorov, A. (2010). Fooled by first impressions? Reexamining thediagnostic value of appearance-based inferences. Journal of Experimental SocialPsychology, 46(2), 315-324.

Roberts, D. J. (2012). Secret ink: Tattoo's place in contemporary American culture. The Journal of American Culture, 35(2), 153-165.

Roggenkamp H, Nicholls A, Pierre J. M. (2017). Tattoos as a window to the psyche: How talking about skin art can inform psychiatric practice. World Journal of Psychiatry. 7(3):148-158. doi:10.5498/wjp.v7.i3.148.

Rubin, H. J., \& Rubin, I. S. (1995). Qualitative interviewing: The art of hearing data. ThousandOaks, CA: Sage.

Ruffle, B., \& Wilson, A. (2017). Tat will tell: Tattoos and time preferences.Rimini Center for Economic Analysis. Available at: http://www.rcea.org/RePEc/pdf/wp18-15.pdf

Ruffle, B. J., \& Wilson, A. (2018). The Truth About Tattoos. Laurier Centre for Economic Research and Policy Analysis. Available at: https://dx.doi.org/10.2139/ssrn.3198397

Salmons, J. E. (2015). Doing qualitative research online. Thousand Oaks, CA: Sage.

Salmons, J. (2017). Getting to Yes: Informed Consent in Qualitative Social Media Research. In The Ethics of Online Research (pp. 109-134). Emerald Publishing Limited.

Sanders, C. R. (1988). Customizing the body: The art and culture of tattooing. Philadelphia, PA: Temple University Press.

Seiter, J. S. \& Hatch, S. (2005). Effect of tattoos on perceptions of credibility and attractiveness. Psychological Report, 96(3), 1113-1120. 
Serup, J., (2015). Seamless prevention of adverse events from tattooing: integrated strategy emphasizing the customer-tattooist interaction. Tattooed Skin and Health. 48, 236-247. Karger Publishers. DOI:10.1159/000370017

Shannon-Missal, L., (2016). Tattoo Takeover: Three in Ten Americans Have Tattoos, and Most Don't Stop at Just One.The Harris Poll. 12.

Sofer, C., Dotsch, R., Wigboldus, D. H., \& Todorov, A. (2015). What is typical is good: The influence of face typicality on perceived trustworthiness. Psychological Science, 26(1), 39-47.

Sperry, K. (1991). Tattoos and tattooing. Part I: History and methodology. The American Journal of Forensic Medicine and Pathology, 12(4), 313-319.

Steele, C. M. (1997). A threat in the air: How stereotypes shapeintellectual identity and performance. American Psychologist,52(6), 613-629.

Swami, V., Stieger, S., Pietschnig, J., Voracek, M., Furnham, A., \&Tovee, M. J. (2012). The influence of facial piercings andobserver personality on perceptions of physical attractiveness and intelligence. European Psychologist, 17, 213-221.

Swami, V., Tran, U. S., Kuhlmann, T., Stieger, S., Gaughan, H., \&Voracek, M. (2016). More similar than different: Tattooed adults are only slightly more impulsive and willing to take risks than Non-tattooed adults. Personality and Individual Differences, 88, 40-44.

Swanger, N. (2006). Visible body modification (VBM): Evidence from human resource managers and recruiters and the effects on employment. International Journal of Hospitality Management, 25, 154-158. doi:10.1016/j.ijhm.2004.12.004

Timming, A. R., Nickson, D., Re, D., \& Perrett, D. (2017). What do you think of my ink? Assessing the effects of body art on employment chances. Human Resource Management, 56(1), 133-149.

Todorov, A., Pakrashi, M., \&Oosterhof, N. N. (2009). Evaluating faces ontrustworthiness after minimal time exposure. Social Cognition, 27, 813-833. doi:10.1521/soco.2009.27.6.813

Totten, J. W., Lipscomb, T. J. \& Jones, M. A. (2009). Attitudes toward and stereotypes of persons with body art: Implications for marketing management. Academy of Marketing Studies Journal. 13(2), 77-96.

Walzer, A., \&Sanjurjo, P. (2016). Media and contemporary tattoo. Communication \& Society, 29(1).

Whorton, R. P. (2015). Marked: A Policy Capturing Investigation of Job Applicant Tattoos as Stigmatizing Marks in Blue and White Collar Employment (Doctoral dissertation, Bowling Green State University).

Wilson, N. H., Wilson, H. A., \& Wilson, M. A. (2017). Tattoos of the Head, Neck and Hands. Primary Dental Journal, 6(3), 56-61.

Wood, T. J. (2014). Exploring the role of first impressions in rater-based assessments. Advances in Health Sciences Education, 19(3), 409-427.

Zestcott, C. A., Bean, M. G., \& Stone, J. (2017). Evidence of negative implicit attitudes toward individuals with a tattoo near the face. Group Processes \& Intergroup Relations, 20(2), 186-201. 